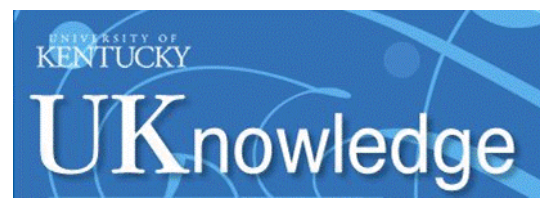

University of Kentucky

UKnowledge

2-10-1995

\title{
On the Current Status of Maser Polarization Theory
}

Moshe Elitzur

University of Kentucky, moshe@pa.uky.edu

Follow this and additional works at: https://uknowledge.uky.edu/physastron_facpub

Part of the Astrophysics and Astronomy Commons, and the Physics Commons

Right click to open a feedback form in a new tab to let us know how this document benefits you.

\section{Repository Citation}

Elitzur, Moshe, "On the Current Status of Maser Polarization Theory" (1995). Physics and Astronomy Faculty Publications. 224.

https://uknowledge.uky.edu/physastron_facpub/224

This Article is brought to you for free and open access by the Physics and Astronomy at UKnowledge. It has been accepted for inclusion in Physics and Astronomy Faculty Publications by an authorized administrator of UKnowledge. For more information, please contact UKnowledge@lsv.uky.edu. 


\section{On the Current Status of Maser Polarization Theory}

\section{Digital Object Identifier (DOI)}

http://dx.doi.org/10.1086/175276

\section{Notes/Citation Information}

Published in The Astrophysical Journal, v. 440, no. 1, p. 345-348.

( $)$ 1995. The American Astronomical Society. All rights reserved.

The copyright holder has granted permission for posting the article here. 


\title{
ON THE CURRENT STATUS OF MASER POLARIZATION THEORY
}

\author{
MOSHE ELITZUR \\ Department of Physics and Astronomy, University of Kentucky, Lexington, KY 40506-0055; \\ moshe@pa.uky.edu \\ Received 1994 April 29; accepted 1994 August 26
}

\begin{abstract}
Despite recent criticism, past conflicts among theoretical studies of maser polarization are by now resolved and clarified. All analytical methods proposed thus far produce the same polarization solutions, solutions that agree with observations. Current numerical studies do not reproduce these solutions. Instead, for any pumping employed, their results are in direct conflict with both analytical theory and observations. These problems reflect the fact that the basic formulation of numerical modeling has yet to incorporate the statistical nature of the radiation field.
\end{abstract}

Subject headings: masers - magnetic fields - polarization - radiative transfer

\section{INTRODUCTION}

Recently, Watson (1994) criticized my work on maser polarization (Elitzur 1991, 1993; hereafter Papers I and II, respectively), proclaiming all of it invalid. Among various technical points, Watson notes that equation (A6) of Paper II is in error, and on this point he is right. Although Paper I lists the correct expressions for $J=1 \rightarrow 0$ absorption coefficients for any type of pumping, those listed in equation (A6) of Paper II for the particular case of $m$-independent pumping are in error. Fortunately, this error has no effect on any of the conclusions of Paper II. The quantities relevant to the discussion of that paper involve differences of absorption coefficients and those are essentially the same. This issue is discussed in $\S 2$ below.

Theoretical papers on maser polarization are inevitably filled with technical details that tend to obscure central issues. Various technicalities notwithstanding, Watson's central assertion is that my work conflicts with every other theoretical study of maser polarization, including the seminal one by Goldreich, Keeley, \& Kwan (1973; GKK hereafter). The principal purpose of this paper is to address this assertion by examining the consistency of all such theoretical studies. A discussion of this issue, free of technicalities to the extent possible, is offered in $\$ 2$ and 3. Some technical details pertinent to Watson's criticism are addressed separately in $\S 4$.

\section{THE CONSISTENCY OF ALL ANALYTICAL SOLUTIONS}

All maser polarization solutions currently known were derived by GKK. They studied radiative transfer of the Stokes parameters $I, Q, U$, and $V$ for $J=1 \rightarrow 0$ masers in four distinct limits, limits defined by two ratios. One is the ratio $J / J_{s}$ of intensity to saturation intensity, the other is the ratio $\Delta v_{B} / \Delta v_{D}$ of Zeeman splitting to Doppler width. Distinctly different methods were proposed for identifying polarizations in the saturated $\left(J / J_{s}>1\right)$ and unsaturated $\left(J / J_{s}<1\right)$ cases. In saturated masers, GKK looked for stationary polarization states, those for which the normalized Stokes parameters $q=Q / I$, $u=U / I, v=V / I$ are constant along the path. With this method, they found independent solutions both for $\Delta v_{B} \gg \Delta v_{D}$ (fully resolved Zeeman pattern) and $\Delta v_{B} \ll \Delta v_{D}$ (overlapping Zeeman components). In the second method, for unsaturated masers, GKK searched for the eigenvectors of the radiative transfer matrix for the four Stokes parameters. For the limit
$\Delta v_{B} \gg \Delta v_{D}$ they found a meaningful eigenvalue equation, whose solution was identical to that for the corresponding saturated maser. However, in the opposite limit, $\Delta v_{B} \ll \Delta v_{D}$, GKK found the eigenvalue problem to be identically diagonal. So their analysis did not identify a polarization solution for unsaturated masers when $\Delta v_{B} \ll \Delta v_{D}$.

Paper I introduced a different approach for identifying polarization solutions, bypassing the radiative transfer equation. Instead, the electric fields of propagating waves were constructed directly, taking into account the constraints imposed by the pump rates and by the transverse condition of Maxwell's equations. The polarization is derived from these explicit vector fields and it reflects their vector structure. Because radiative transfer is not a consideration, the degree of saturation is not a factor. This technique reproduces the independent polarization solutions that GKK found in the limits $\Delta v_{B} \gg \Delta v_{D}$ and $\Delta v_{B} \ll \Delta v_{D}$ and shows that they apply to all spins. It explains for the first time why GKK obtained the same result for saturated and unsaturated masers when $\Delta v_{B} \gg \Delta v_{D}$. It shows that the same applies also in the opposite limit, $\Delta v_{B} \ll$ $\Delta v_{D}$, even though GKK could not identify a solution for such masers when they are unsaturated.

The two analytical studies produced the same results in three relevant limits but reached different conclusions in the fourth, that of unsaturated masers when $\Delta v_{B} \ll \Delta v_{D}$. This difference was resolved in Paper II. Stationary polarization, the condition used by GKK for saturated masers, implies

$$
q^{\prime}=u^{\prime}=v^{\prime}=0,
$$

where a prime denotes derivative along the path. But $q=Q / I$, so $q^{\prime}=0$ is equivalent to $Q^{\prime} / Q=I^{\prime} / I$, with a similar relation for $U$ and $V$. Therefore, the previous equation is equivalent to

$$
\frac{I^{\prime}}{I}=\frac{Q^{\prime}}{Q}=\frac{U^{\prime}}{U}=\frac{V^{\prime}}{V} \equiv \lambda,
$$

where $\lambda$ is some (unknown) factor, common to all four Stokes parameters. That is, stationary polarizations are the solutions of the eigenvalue problem, the GKK technique for unsaturated masers. The two GKK methods are equivalent - every solution of either one of them is also a solution of the other. Therefore, the polarization solution GKK identified in the limit $\Delta v_{B} \ll \Delta v_{D}$ for saturated masers applies also when such masers are unsaturated, in agreement with Paper I. 
Since the two GKK methods are equivalent, why did their analysis not produce the unsaturated maser solution when $\Delta v_{B} \ll \Delta v_{D}$ ? At the time, general results for polarized line radiative transfer were not available, and in each of the four relevant limits GKK had to derive expressions starting anew from Maxwell's equations. The procedure is tedious and the expressions are quite involved. Fortunately, Litvak (1975) has performed an elegant analysis whose general results are more transparent. Each $\Delta m$ transition is characterized by an absorption coefficient $\kappa^{\Delta m}$. Because of the assumption $\Delta v_{B} \ll \Delta v_{D}$ and the symmetry around the magnetic axis, $\kappa^{+1}=\kappa^{-1} \equiv \kappa^{1}$ and there are only two independent coefficients, $\kappa^{0}$ and $\kappa^{1}$, listed in equation (5.27) of Paper I. With the additional assumption of equal pump and loss rates for the magnetic sublevels $(\Delta m$ independent $J_{s}$ and unsaturated absorption coefficient $\kappa_{0}$ ), these absorption coefficients are

$$
\kappa^{0}=\kappa_{0}\left(1+f^{1} J / J_{s}\right) / D, \quad \kappa^{1}=\kappa_{0}\left(1+f^{0} J / J_{s}\right) / D,
$$

where

$$
D=1+\left(2 f^{0}+3 f^{1}\right) J / J_{s}+4 f^{0} f^{1}\left(J / J_{s}\right)^{2} .
$$

These expressions, which are in agreement with those used by Watson (1994), replace the erroneous equation (A6) of Paper II. The factors $f^{\Delta m}$, which vary between 0 and 1 and are listed in GKK (eqs. [46]) and in Paper II (eq. [A7]), depend only on the normalized Stokes parameters and on the angle $\theta$ between the magnetic field and the propagation direction. Under these circumstances, the radiative transfer matrix has only one offdiagonal element.

$$
\kappa_{l}=\frac{1}{2}\left(\kappa^{1}-\kappa^{0}\right) \sin ^{2} \theta=\frac{1}{2} \kappa_{0} \sin ^{2} \theta\left(f^{0}-f^{1}\right) J /\left(J_{s} D\right) .
$$

The expression in Paper II for $\kappa_{l}$ is the same, except that it has $D=1+\left(f^{0}+f^{1}\right) J / J_{s}+f^{0} f^{1}\left(J / J_{s}\right)^{2}$. The unsaturated limit, which was used extensively in Paper II, is the same for both expressions since $D=1$ in either case. The transfer matrix becomes diagonal when $\kappa_{l}$ vanishes, i.e., when

$$
0=f^{1}-f^{0}=\frac{3}{4} \sin ^{2} \theta\left[q-q_{p}(\theta)\right],
$$

where the last equality follows from the explicit expressions for $f^{1}$ and $f^{0}$. Here $q_{p}(\theta)=1-2 /\left(3 \sin ^{2} \theta\right)$ is the GKK polarization solution for $\theta \geq \theta_{0}$, where $\sin ^{2} \theta_{0}=\frac{1}{3}$ (this solution is unphysical for $\theta<\theta_{0}$, where $\left.\left|q_{p}(\theta)\right|>1\right)$. Matrix diagonalization produces the GKK polarization solution independent of the saturation stage, in agreement with Paper I. Another solution, $q^{2}=1$, is obtained from a closer examination of the explicit eigenvalue problem.

Without the benefit of Litvak's formalism, GKK derived the radiative transfer equations for each case after first expanding $\kappa^{\Delta m}$ to leading order in the relevant parameter- $J / J_{s}$ for unsaturated masers, $J_{s} / J$ for saturated ones. As is evident from equations (3) and (4), to leading order $\kappa_{l}$ is linear in the expansion parameter in either regime. However, the leading order terms of $\kappa^{\Delta m}$ produce this result only for saturated masers. When $J / J_{s}<1, \kappa^{\Delta m}$ must be expanded to the next to the leading order to produce the correct $\kappa_{l}$. At the leading order $\kappa^{0}=\kappa^{1}=\kappa_{0}$, resulting in $\kappa_{l}=0$ independent of, and without any meaningful constraint on, the polarization. This is the reason why this solution was originally missed.

As this discussion shows, the only difference between the conclusions of GKK and Paper I has been fully resolved. The two studies are in complete agreement.

\section{PROBLEMS WITH EXISTING NUMERICAL SOLUTIONS}

The other studies of the maser polarization problem were reported in a series of papers by Watson and various collaborators (e.g., Western \& Watson 1984; Deguchi, Watson, \& Western 1986; Nedoluha \& Watson 1990). These studies considered only $\Delta v_{B} \ll \Delta v_{D}$ and followed the growth of the Stokes parameters numerically, starting from zero polarization. According to these calculations, the GKK solution for a $J=1 \rightarrow 0$ transition is reached only as a limit for $J / J_{s} \gtrsim$ $10^{2}-10^{3}$, values much higher than observations suggest. Since the only analytical study available at that time was GKK's, this numerical result had an explanation: GKK obtained a solution for $\Delta v_{B} \ll \Delta v_{D}$ only in the saturated regime, so one might expect this polarization to be approached only as a limit when $J>J_{s}$ (though $J>10^{2} J_{s}$ is surprising). However, as was just shown, the eigenvalue solutions are independent of $J / J_{s}$ whatever the relation between $\Delta v_{B}$ and $\Delta v_{D}$; thus they are not merely limit solutions. So the numerical results conflict with the analytical solutions.

Another problem is posed by observations. The numerical solutions found that higher spin transitions do not reach the GKK solution at any intensity, have polarization characteristics different from $J=1 \rightarrow 0$, and possess progressively less fractional polarization as the spin increases. These results are especially relevant to $\mathrm{SiO}$, which has a number of consecutive rotation state masers, and Nedoluha \& Watson (1990) concluded that these masers must originate in different regions. This conclusion was based on calculations that considered both isotropic ( $m$-independent) and anisotropic ( $m$-dependent) pumping; both types of pumping led to similar predictions. Attempting to verify these predictions, McIntosh \& Predmore $(1991,1993)$ observed the SiO lowest three rotation transitions in a number of late-type stars. Rather than decrease, the fractional polarization was found to increase monotonically with the transition spin. The $J=3 \rightarrow 2$ transition is the most strongly polarized, with a polarization that approaches $100 \%$ in Mira. Similarities in detailed polarization properties among different transitions indicate that they all originate in the same volume of gas. On every point, the observational results are the opposite of what the numerical solutions predict. In contrast, these findings are those expected if linear polarization is produced as described in Papers I and II in the presence of free electrons. Then the GKK solution is reached for all spins, so different masers can be inverted in the same location and have similar linear polarization, as high as $100 \%$. Subsequent Faraday depolarization affects the lower spin states more because of their longer wavelengths - the observed polarization increase toward higher spins is actually a decrease toward lower ones because of Faraday depolarization. Although detailed calculations of Faraday rotation have yet to be performed, McIntosh \& Predmore (1993) find this to be the most plausible explanation of their data.

Water masers exhibit similar discrepancies with the numerical results. The flare in Orion had high linear polarization during its decay (Abraham 1994), the opposite of the trend predicted by all numerical models. These discrepancies pose a serious problem. Analytical analysis of the radiative transfer equations produces a unique stationary solution consistent with observations, yet a numerical solution of these very equations does not reproduce this result. This conflict can be traced to a fundamental ingredient missing from the formulation of the numerical problem, identified in Paper II to be the sta- 
tistical nature of the radiation field. The radiation field is an ensemble of waves. According to Maxwell's equations, each wave is fully polarized and the observed polarization is the average over all the modes actually present in the field. Similar to thermal motions, each mode is characterized by $S$, the 4-vector of its Stokes parameters, and the statistical mode distribution at position $l$ is described by a function $\mu(S, l)$, normalized such that $\int \mu(S, l) d S=1$. The observed Stokes parameters are the ensemble averages

$$
\langle\boldsymbol{S}(l)\rangle=\int \boldsymbol{S} \mu(\boldsymbol{S}, l) d \boldsymbol{S} .
$$

In principle, such averages could be obtained observationally from an ensemble of many maser sources with identical physical properties. In practice, the Stokes parameters are obtained from time averages of the observations of a single source. If time sampling could be performed so frequently and with such angular resolution as to detect the arrival of individual waves, the recorded radiation would always be fully polarized, although time-variable, and the actual polarization is the time average of those different values.

The task for theory is to calculate the observable $\langle\boldsymbol{S}\rangle$. This is precisely what is accomplished by the solutions of the eigenvector problem, for the following reason: These solutions are determined by the functional forms of the absorption coefficients; for example, in the $\Delta v_{B} \ll \Delta v_{D}$ case the solution follows from $\kappa^{0}=\kappa^{1}$. The absorption coefficients are determined by the level populations in steady state, which in turn reflect the various particle interactions, including interactions with the maser radiation. Because particles interact with radiation that travels in any direction and has different polarization properties, the radiative terms in the rate equations involve averages over all the properties of the local radiation field, analogous to the averages over particle velocities of the collision terms. This is the reason why the radiative rates always contain the angleaveraged intensity $J$, and when polarization is included they are also averaged over all the modes present in the radiation field. Since the radiative dependence of the absorption coefficients involves the ensemble-averaged Stokes parameters, these are the quantities that the eigenvector solutions determine.

Whereas Paper II only recognized the role of an eigenvector as the peak of the appropriate polarization mode distribution, this discussion shows that the eigenvectors are also the distribution averages, i.e., the observed quantities. When $\Delta v_{B} \gg$ $\Delta v_{D}$, the stationary solutions apply already at the source function level because only one Zeeman component is generated at a given frequency. When $\Delta v_{B} \ll \Delta v_{D}$, Paper II shows that maser amplification is accompanied by rotation of each polarization vector toward the stationary mode at a rate proportional to $q-q_{p}(\theta)$. While different modes follow different growth paths, when $\theta>\theta_{0}$ they all evolve toward the same mode $q=q_{p}(\theta)$, the GKK polarization solution, so the average over all modes (the observed quantity) can reach this value before individual modes do. The solution is applicable once the terms associated with self-amplification dominate the radiative transfer, a stage reached after unsaturated amplification with $\tau \sim 6-9$. This marks the onset of the polarization solution for all spins, a conclusion supported by observations.

Wherever applicable, the eigenvalue method identifies the ensemble averages from local properties. In contrast, the goal of numerical modeling is to follow the evolution of these averages along the radiation path, a considerably more challenging task. In addition to the evolution of the Stokes parameters of individual modes, the mode distribution $\mu(S, l)$ can evolve too and both variations must be included in a calculation of $d\langle\boldsymbol{S}(l)\rangle / d l$. The situation is greatly simplified when all modes grow at the same rate. Then the mode distribution is constant along the path $(d \mu / d l=0)$, the evolution of the ensemble averages is identical to that of any individual mode and the mode distribution need not even be specified. This is the situation in the scalar maser model, the widely used approximation that treats the radiation electric field as a scalar (one intensity and one absorption coefficient). In the unsaturated regime, where growth is exponential, the growth rate of scalar maser intensity is $\kappa_{0}$, independent of intensity to leading order. Therefore, the scalar unsaturated maser is a purely linear amplifier; its growth rate is independent of intensity, i.e., it is modeinvariant. Every mode grows at the same rate and any intensity spectrum is amplified without distortion (as long as it is not dominated by fluctuations so extreme as to induce premature saturation). This is the reason why the need to consider ensemble averages usually does not arise for scalar masers.

The inclusion of polarization changes the problem fundamentally. A general, formal solution presented in Paper II shows that in the unsaturated regime, the Stokes parameters contain a term $\exp \left[\int\left|a\left(q-q_{p}\right)\right|\left(J / J_{s}\right) \kappa_{0} d l\right]$, where $a$ is of order unity; the exponential growth rate depends on fractional polarization and intensity at the leading order. Since the growth rates of the Stokes parameters depend on the Stokes parameters themselves, the polarized unsaturated maser is a nonlinear amplifier. Every mode grows at a different rate, i.e., the growth rate is mode-dependent, so the mode distribution does get modified during unsaturated amplification. An effect that occurs in scalar masers only during extreme, violent fluctuations, is an integral part of polarization growth and a calculation of the growth of ensemble-averages must account for the evolution of $\mu(S, l)$ along the path. This requires simulations that treat the radiation field as a statistical ensemble of waves evolving from an initial distribution. Such simulations are frequently performed in studies of plasma and laboratory lasers, but have not yet been attempted in published numerical studies of astronomical maser radiation. Instead, the available calculations effectively describe the evolution of a radiation field in which all waves are launched with a single intensity (the source function) and $q=0, u= \pm 1, v=0$. The evolution of this singular configuration is not indicative of the actual, observed polarization just as the dynamic behavior of a collection of particles that start with zero velocity is not representative of finite temperature gas even though the velocity averages of both ensembles are the same.

\section{INSTABILITIES}

Watson (1994) presents a formal stability analysis, similar to one performed in Paper II for unsaturated masers when $\Delta v_{B} \ll$ $\Delta v_{D}$. He concludes that maser radiation is stable for "all relevant" propagation directions and initial polarizations. Although a precise definition of "all relevant" is not given, ${ }^{1}$

\footnotetext{
${ }^{1}$ Watson's conclusion could be interpreted to imply that there exists some finite region of phase space in which all modes are stable. That is, a perturbation around any configuration in that region would always evolve toward the initial state. Such a possibility is excluded on general grounds. Consider two different nearby states $A$ and $B$ in such a region of phase space, if it were to exist. If $B$ is considered a perturbation of $A$, then $B$ should evolve toward $A$, yet if $\mathbf{A}$ is considered a perturbation of $B$, then $A$ should evolve toward $B$.
} 
Watson states that his result contradicts Paper II. This issue is briefly addressed here.

Beginning with GKK, stability analysis was employed to identify physical polarization when more than one eigenvector solution existed by eliminating unstable ones. In practice, this technique was only required for $\Delta v_{B} \leqslant \Delta v_{D}$. Stability analyses for this case were performed also by Western \& Watson (1984) and in Paper II. For propagation at $\theta \geq \theta_{0}$, all studies are in agreement that $q=q_{P}(\theta)$ is the only stable eigenmode. But for $\theta<\theta_{0}$, Paper II concludes that the eigenmode $q=-1$, thought to be stable, is actually unstable during the previously ignored unsaturated growth phase. Since $\theta<\theta_{0}$ comprises less than $10 \%$ of space and involves fully polarized radiation which is rarely observed in nonparamagnetic masers, setting aside this disputed region has little impact on the comparison with observations.

Another issue is stability analysis for nonstationary modes. As shown in the previous section, a key property in controlling ensemble averages of polarized maser radiation is that, in the unsaturated regime, the exponential growth rates of the Stokes parameters depend on the Stokes parameters themselves. The significance of this behavior was first noted in Paper II, which referred to it as "instability." Although technically correct, this was the wrong concept to invoke: since every mode grows at a different rate, configurations belonging to different modes diverge exponentially away from each other however close they initially are, a behavior generally termed "instability." However, exponential growth in masers is the very essence of the effect itself and follows directly from the equation of radiative transfer without stability analysis perturbations. Indeed, such analysis cannot be performed unambiguously around anything other than eigenmodes. These are the only modes characterized by pure exponential growth, the evolution of all other modes involves combinations of exponential terms with different growth rates. Therefore, the removal of a base solu- tion around which to perturb is ill defined for nonstationary modes. This could explain possible differences between the conclusions of Watson (1994) and Paper II. However, this issue is now moot. As shown here, the essence of nonlinear amplification is the variation of the mode distribution, not instabilities.

\section{SUMMARY}

Different analytical methods produce the same solutions for the maser polarization problem, both when $\Delta v_{B} \ll \Delta v_{D}$ and when $\Delta v_{B} \gg \Delta v_{D}$. In both limits, the solutions adequately describe all available observations, though Faraday rotation has yet to be properly incorporated to explain some details. Thus far, numerical studies for $\Delta v_{B} \ll \Delta v_{D}$ have failed to reproduce the analytical solution, instead they produced results that directly conflict with both analytical results and observations. This failure reflects deficiencies in basic formulation which does not incorporate the statistical nature of the problem.

Proper numerical simulations are an important task for future studies. For any statistical distribution, it is always much easier to identify the stationary limit than to demonstrate how that limit is actually approached; a demonstration of the approach to Maxwellian of a particle velocity distribution or to Planckian of a photon distribution are considerably more difficult than the derivation of either functional form. How the ensemble averages of maser radiation fields evolve toward the eigenvalue solutions from various initial mode distributions is an important problem. Ultimately, its complete solution will require numerical simulations that follow the evolution of an amplified radiation field properly described as a statistical ensemble.

This work was supported by NSF grants AST-9016810 and AST-9321847.
Abraham, Z. 1994, A\&A, in press

Deguchi, S., Watson, W. D., \& Western, L. R. 1986, ApJ, 302, 108

Elitzur, M. 1991, ApJ, 370, 407 (Paper I) 1993, ApJ, 416, 256 (Paper II)

Goldreich, P., Keeley, D. A., \& Kwan, J. Y. 1973, ApJ, 179, 111 (GKK)

Litvak, M. M. 1975, ApJ, 202, 58

\section{REFERENCES}

McIntosh, G., \& Predmore, C. R. 1991, in Skyline: Proc. 3rd Haystack Conf., vol. 16, ed. A. D. Haschick \& P. T. P. Ho (ASP Conf. Ser., 16), 83

McIntosh, G., \& Predmore, C. R. 1993, ApJ, 404, L71

Nedoluha, G., \& Watson, W. D. 1990, ApJ, 354, 660

Watson, W. D. 1994, ApJ, 424, L37

Western, L. R., \& Watson, W. D. 1984, ApJ, 285, 158 